\title{
Exploring structure and function of sensory cortex with 7 T MRI
}

Special issue: Spatio-temporal limits

Denis Schluppeck ${ }^{1, *}$

Rosa-Maria Sanchez-Panchuelo²

Susan T Francis ${ }^{2}$

${ }^{*}$ Corresponding author:

denis.schluppeck@nottingham.ac.uk

t: +441158468580

${ }^{1}$ School of Psychology

${ }^{2}$ Sir Peter Mansfield Imaging Centre, School of Physics and Astronomy,

University of Nottingham

University Park, Nottingham, NG7 2RD, UK

Number of pages:

Number of figures, table

Number of words for Abstract, Introduction, and Discussion (separately)

Conflict of Interest: None

Acknowledgements: We would like to thank Keren Yang, Jack Harmer and Julien Besle for help with software, data acquisition, and analysis.

Author contributions: DS, RM and STF designed experiments, analyzed data and wrote the paper. STF gratefully acknowledges funding from the MRC (MR/M022722/1) for the TOUCHMAP project. 


\section{Abstract (max 250w)}

In this paper, we present an overview of 7 Tesla magnetic resonance imaging (MRI) studies of the detailed function and anatomy of sensory areas of the human brain. We discuss the motivation for the studies, with particular emphasis on increasing the spatial resolution of functional MRI (fMRI) using reduced field-of-view (FOV) data acquisitions. MRI at ultra-high-field (UHF) - defined here as

$7 \mathrm{~T}$ and above - has several advantages over lower field strengths. The intrinsic signal-to-noise ratio (SNR) of images is higher at UHF, and coupled with the increased blood-oxygen-level-dependent (BOLD) signal change, this results in increased BOLD contrast-to-noise ratio (CNR), which can be exploited to improve spatial resolution or detect weaker signals. Additionally, the BOLD signal from the intra-vascular (IV) compartment is relatively diminished compared to lower field strengths. Together, these properties make $7 \mathrm{~T}$ functional MRI an attractive proposition for high spatial specificity measures. But with the advantages come some challenges. For example, increased vulnerability to susceptibility-induced geometric distortions and signal loss in EPI acquisitions tend to be much larger. Some of these technical issues can be addressed with currently available tools and will be discussed. We highlight the key methodological considerations for high resolution functional and structural imaging at $7 \mathrm{~T}$. We then present recent data using the high spatial resolution available at UHF in studies of the visual and somatosensory cortex to highlight promising developments in this area.

\section{Highlights (max 120w)}

- Magnetic resonance imaging, MRI, at $7 \mathrm{~T}$ can provide increased BOLD contrast-to-noise ratio compared to lower field strengths

- For many neuroscience applications, $\mathrm{fMRI}$ at $7 \mathrm{~T}$ has clear advantages: (a) with voxel sizes of 1 $\mathrm{mm}^{3}$ and below, spatially resolving small brain structures becomes possible; (b) measuring weaker signals such as biases across cortical maps in individual participants is easier; (c) the sensitivity of functional signals from methods other than gradient-echo EPI based BOLD contrast become more robust and usable.

- We show recent results of mapping somatosensory and visual areas with a reduced field-of-view (FOV) at high spatial resolution, and summarize methodological advances in using these methods. 


\section{Introduction ( 300w)}

In recent years there has been increased attention for the use of magnetic resonance imaging at ultra-high field, field strengths of 7 Tesla and above. With the expanding availability of ultra-high-field (UHF) MRI, a growing number of studies have demonstrated the benefits of this technology, particularly for high spatial resolution functional and structural measurements.

The history of imaging at ultra-high field, and a detailed discussion of the advantages have been presented elsewhere (Harel 2012, Ugurbil 2012, Budinger, Bird et al. 2016). The aim of this paper is to provide a brief overview of the $7 \mathrm{~T}$ studies exploiting high resolution functional and structural measures, referring to reports in the literature and recent data collected from our group on the Philips Achieva 7 Tesla scanner at the University of Nottingham. In our hands, the advantage of UHF fMRI has largely been used in targeted studies with partial coverage of the brain. We show how a combination of techniques can be used to study detailed function and anatomy of the somatosensory and visual cortex. We also demonstrate how the techniques and analysis methods we have developed for measuring the cortical representation of touch may be applied to the study of other functional areas in the brain, for example the auditory cortex.

Our general approach across these studies has been to use a set of tractable neuroscience questions to validate our approach and build confidence in novel methodology. For example, we measure the representation of the skin surface in human primary somatosensory cortex (S1) to form somatotopic maps. Importantly, we typically repeat our scanning sessions across a small pool of participants, as a model system to develop our acquisition techniques and analysis methods, and test the reproducibility with which individual subject analysis can be achieved at UHF.

\section{$M R I$ at 7 T provides better signal and contrast}

The improvements in SNR and CNR afforded by imaging at UHF can be used to increase spatial or temporal resolution - in this paper we focus on the application to high spatial resolution. Smaller voxels allow more faithful measurement of the layout of structures which may be below the sampling limit of larger voxels sizes and therefore aliased. Second, the reduction in partial volume effects with smaller voxel sizes compensates for reduction in signal strength with volume. Combined with experimental paradigms that preferentially drive input and output layers of the cortex, acquisition at higher spatial resolution allows the non-invasive study of the brain at the scale of cortical columns and layers (De Martino et al 2013).

The increased BOLD CNR of UHF experiments also allows the reduction in number of repeated measurements while still allowing for reliable measurements of functional responses in the brain. Traditionally, event-related experiments rely on signal averaging over many trials or using repeated trials for fitting models. For example, in our initial event-related experiments to measure cortical responses to vibrotactile stimulation of each of the fingertips and the overlap of cortical 
representations, we used > 30 trials per condition for generalized linear model (GLM) or deconvolution analyses (Besle, Sanchez-Panchuelo et al. 2013, Besle, Sanchez-Panchuelo et al. 2014). By combining data across many trials in which the signals are consistent, but unwanted contributions from noise are uncorrelated, such signal averaging allowed robust estimation of responses. The same logic also applies for other data analysis approaches such as the population receptive field (pRF) method (Dumoulin and Wandell 2008), which relies on non-linear least-squares optimization. The limit of this approach is to measure fMRI responses locked to single sensory or cognitive events. An alternative way to exploit the SNR and CNR improvements is to increase temporal resolution. Recent developments in this area are discussed elsewhere in this special issue. We first provide a brief summary of our approaches to combine high spatial resolution functional with detailed quantitative, structural data at $7 \mathrm{~T}$. We then outline recent applications of these techniques to the study of human somatosensory and visual cortex. We discuss key findings and how we believe this methodology can be applied to map other cortical areas. In our studies, we were particularly interested in determining the detailed function and anatomy in individual subjects with a view to translational and potentially clinically applications.

\section{Methodological considerations}

Here we concentrate on increasing the spatial resolution of functional MRI studies. In these experiments, high spatial resolution measurements have been typically confined to a small field of view of the brain and we have generally used BOLD contrast based on gradient-echo (GE) measures. In the following, we briefly outline some observations that proved especially important for our studies. Most of these points apply to UHF experiments in general, although some are more specific to the hardware we used (7 T, Philips Achieva).

\section{[[ Figure 1 about here - methods ]]}

\section{Distortion correction}

Good correction of inhomogeneities in the static magnetic field, $B_{0}$, is crucial for high quality data. In EPI acquisitions, inhomogeneities in the local magnetic field can lead to geometric distortions. We use local image-based (IB) shimming for all fMRI acquisitions (Wilson, Jenkinson et al. 2002, Poole and Bowtell 2008), and have shown that over a restricted FOV of interest (such as the somatosensory cortex and occipital lobe) this reduces field perturbations to typically $<20 \mathrm{~Hz}$. 
By combining IB shimming with a reduced FOV data acquisition, it is possible to restrict geometric distortions to less than one voxel $(<1 \mathrm{~mm})$ in GE-EPI data. For example, our restricted FOV, $1 \mathrm{~mm}$ $\mathrm{fMRI}$ data acquired on the somatosensory cortex using the 32-channel NOVA receive coil typically uses a $72 \mathrm{~mm}$ FOV in the right-left $(R L)$ phase encoding direction combined with outer volume suppression (Pfeuffer et al. 2002) to prevent signal fold-over. With a $1500 \mathrm{~Hz}$ bandwidth in the read direction, this results in an acquisition of $\sim 0.7 \mathrm{~ms}$ per line. Coupled with a SENSE reduction of 2.5 this yields $\sim 20 \mathrm{~Hz}$ bandwidth in phase encoding direction. Alternatively, for higher spatial resolution $(<1 \mathrm{~mm})$ somatosensory data acquisitions, as described below, we use a local surface receive coil which allows increased SENSE reduction, typically with a factor of $\sim 4$, again resulting in a bandwidth in the phase encoding direction of $\sim 20 \mathrm{~Hz}$. For all high spatial resolution $\mathrm{fMRI}$ studies, we generally collect data across repeat fMRI runs, and for this it is essential to keep the shim settings fixed throughout the duration of the scanning session, rather than estimating the shim at the beginning of each $\mathrm{fMRI}$ run. Even though small residual inhomogeneities will be present, the geometric distortions in the reconstructed fMRI data are largely stable across the scanning session.

The use of a reduced field of view (FOV) to zoom into a local cortical region of interest to yield high spatial resolution $\mathrm{fMRI}$ also provides other advantages. For example, the implementation of a full FOV high spatial resolution EPI acquisition results in long echo train lengths which limit achieving the echo time required to optimize BOLD contrast (grey matter $\mathrm{T}_{2}{ }^{*}$ of $\sim 25-30 \mathrm{~ms}$ at $7 \mathrm{~T}$ ). In zoomed EPI acquisitions, the FOV in the phase encoding direction can be reduced using outer suppression rest slabs to prevent fold-over artefacts, thus decreasing image acquisition time and removing any limitations on achieving the optimal echo time.

In addition to minimizing any residual distortion in the acquisition, we routinely also collect data such that - if required - we can perform static distortion correct of the data, using either a conventional dual echo-time field mapping (Hutton, Bork et al. 2002) or by acquiring a data set with opposing phase encoding directions to estimate the field and correct for distortions (Andersson, Skare et al. 2003), as has been made available by FSL via a tool called TOPUP ( http://fsl.fmrib.ox.ac.uk/fsl/fslwiki/topup ).

In recent studies, we go beyond assuming that the $B_{0}$ inhomogeneities estimated at a given time in a scanning session are the same throughout, and have made use of dynamic $B_{0}$ field correction, in which we estimate - and correct for - slightly changing $B_{0}$ inhomogeneities at each point in the acquired time series. Details of this method have been presented previously in abstract form (Harmer, Francis et al. 2011). 
Functional imaging based on GE BOLD contrast is a sensitive and versatile technique, For high spatial resolution studies requiring a large number of slices, 3D GE-EPI provides an attractive alternative to standard 2D GE-EPI, as the SNR of 3D GE-EPI acquisitions improves with increasing number of slices (Poser, Koopmans et al. 2010), and parallel imaging allows acceleration to be applied in two spatial dimensions, significantly reducing the total volume acquisition time (Barry, Strother et al. 2012, van der Zwaag, Kusters et al. 2012). However, it is possible to shorten the dynamic scan time of 2D-EPI sequences, while maintaining high spatial resolution, using multiplex techniques (Simultaneous Multi-Slice (SMS) or Multiband imaging) where multiple slices are simultaneously excited and sampled (Feinberg, Moeller et al. 2010, Moeller, Yacoub et al. 2010).

An alternative to gradient echo based $f M R I$ is to obtain BOLD contrast using spin echo (SE) $T_{2^{-}}$ weighted imaging. This has the advantage that it is less sensitive to signals arising from large veins. However, this comes at the cost of reduced sensitivity (Norris 2012). For SE BOLD imaging, the improved spatial accuracy is best realised at UHF due to the reduced contribution of the intravascular component to the overall BOLD contrast. UHF $7 \mathrm{~T}$ allows measurements of sequences that lack sensitivity at $3 \mathrm{~T}$ or below. For example, BOLD measurements with $\mathrm{T}_{2}$-weighted spin-echo sequences eliminate the extravascular (EV) contribution from large vessels and are particularly challenging at lower field strength (Norris 2012). UHF provides improved sensitivity to signal changes around small venules and capillaries. Because of improved spatial specificity, SE BOLD has been used to map cortical columns in humans (Yacoub, Shmuel et al. 2007, Yacoub, Harel et al. 2008) as well as cortical layer specific activity (Olman, Harel et al. 2012, De Martino, Zimmermann et al. 2013, Siero, Ramsey et al. 2013).

Although it is thought to be less spatially specific than SE BOLD (Norris 2012), there are many instances when GE BOLD is the best choice for experiments, due to its inherent increased BOLD contrast. We used a dual GE-SE acquisition scheme in which acquisitions of both GE and SE BOLD were simultaneously collected (within a few milliseconds of each other) to measure activity in bands in the primary visual cortex induced using visual stimuli comprising of complementary rings of contrast-reversing checkerboard patterns $(8 \mathrm{~Hz})$. Our data showed that for structures larger than $\sim 1.5 \mathrm{~mm}$ in size GE BOLD was preferable over SE BOLD. In this experiment, the use of a differential paradigm (block-alternation between two similar stimulus conditions) may also have helped to reduce larger transient signals in larger draining veins that can occur with more sparsely timed stimulus events (Sanchez Panchuelo, Schluppeck et al. 2015).

MRI measures sensitive to functional changes in cerebral blood volume (CBV) or cerebral blood flow (CBF) provide alternative methods which also offer improved spatial specificity over standard BOLD. Although the sensitivity of these modalities is lower than GE BOLD, CBV and CBF measures are believed to be more closely related to micro-vessels within layers, which may be preferable for layer 
specific studies. Dynamic susceptibility contrast agents have been used to enhance the sensitivity of measures of $\mathrm{CBV}$ changes, but recently it has also been shown that non-invasive strategies based on vascular space occupancy (VASO) can detect CBV changes with high CNR in the human brain at 7T (Huber, Ivanov et al. 2014), and capture cortical layer-dependent activity changes (Huber, Goense et al. 2015).

In addition to the considerations regarding imaging parameters and techniques, there are a host of other considerations related to data analysis and experimental design. For measurements at the columnar and layer level, for example, cortical segmentation and flattening to allow faithful visualization become increasingly important. The details of these, however, are beyond the scope of this paper.

\section{High spatial resolution mapping of somatosensory cortex}

From direct electrical stimulation of the human cortex, there is clear evidence - at a coarse level that the digits of the hand are mapped on the post-central gyrus in an area overlapping cytoarchitectonic fields of areas 3a, 3b, 1, and 2 (Penfield and Boldrey 1937). Cortical responses to tactile events have been difficult to measure using conventional fMRI experiments. In particular, the reliable localisation and subdivision of primary somatosensory cortex (S1) has been hard to achieve below 7 T (Francis, Kelly et al. 2000, Nelson and Chen 2008, Overduin and Servos 2008). A series of experiments from our group has demonstrated how reliable maps of finger representations of individual subjects can be achieved at $7 \mathrm{~T}$ (Sanchez-Panchuelo, Francis et al. 2010). These measurements are stable across sessions and are largely unaffected by the details of the acquisition protocol (Figure 2).

We have also demonstrated that it is possible to measure the finer-scale sub-divisions of $\mathrm{S} 1$, using within-digit organization (base-to-tip) stimulation of digits, providing functional parcellation of Brodmann areas of human S1 (Sanchez-Panchuelo, Besle et al. 2012). We have assessed the relationship between these functional domains and underlying structure by inspecting our functionally parcellated Brodmann areas for differences in cortical thickness and alternative MR contrast measures. We evaluated both magnetization transfer ratio (MTR) and signal intensity in phase sensitive inversion recovery (PSIR) images that are sensitive to the pattern of myelination (Sanchez-Panchuelo, Besle et al. 2014). In this study, we showed consistent, area-related differences in cortical thickness and MTR/PSIR measurements across subjects. A key aspect to this work was the increased sensitivity of functional MRI at UHF that enabled very high spatial resolution measurements of the functional architecture of individual human brains. By combining the technique of intraneural microstimulation (INMS) with UHF FMRI, we have recently mapped the cortical responses elicited by single mechanoreceptive afferent units in the median nerve (Sanchez Panchuelo, Ackerley et al. 2016). Together, these techniques make an attractive set of tools for basic neuroscience and clinical applications. 


\section{[[ Figure 2 about here - somatosensory maps ]]}

Now, with the use of local surface receive coils, we can begin to improve the spatial resolution of these measures such that robust maps can be obtained for sub-millimetre voxel volume (Figure 3). This developments will be used to begin to address the cortical layer-dependent activity in response to tactile stimulation of the digits, and to resolve the cortical responses from different types of individually characterized mechanoreceptive afferents.

\section{[[ Figure 3 about here -coils ]]}

\section{High-resolution retinotopy and orientation mapping - visual cortex}

The function and detailed organization of visual cortex is a topic of great interest to a large community of neuroscientists, past and present. For studying the human visual system, a combination of psychophysics and functional MRI has become a dominant technique (see Bandettini 2012). Because visual stimuli can be under excellent experimental control and because a lot is known about the anatomy and neurophysiology of the visual system in several species (including non-human primates and other mammals), the visual cortex also provides an ideal framework for pushing improvements in imaging methodology.

In particular, the retinotopic organization of early visual areas is particularly well understood and there is a rich literature connecting experiments in non-human primates to detailed measurements with fMRI (for a review of visual field maps see Wandell, Dumoulin et al. 2007). The ability to locate primary visual cortex, V1, and neighboring visual areas (V2, V3, etc.) in vivo, based on both anatomical and functional criteria, has provided an important starting point for researchers interested in measuring the columnar and layer organization of cortex.

Strong predictions on the layout and scale of columns that should be observed with functional MRI come from extensive studies in non-human primates and in the case of ocular dominance columns, also from human post-mortem samples (Mountcastle 1997, Adams, Sincich et al. 2007). Several studies from the Minnesota group have demonstrated the approach for direct measurements of the columnar organization for ocular dominance and orientation (Yacoub, Shmuel et al. 2007, Yacoub, Harel et al. 2008).

But there is also a large body of work that has aimed to measure functional organization at the columnar level indirectly, using multivariate statistical analysis and machine learning methods. Starting with work of Kamitani and Tong (2005) and Haynes and Rees (2005), many of these studies 
have hypothesized that slightly uneven sampling of the cortical columnar architecture introduces small biases in the pattern of responses across many voxels.

Here, we measured responses in the visual cortex to two kinds of visual stimuli used in such studies, allowing us to evaluate our image acquisition and data analysis protocol. We were motivated by a previous study (Freeman et al $2010 \mathrm{~J}$ Neursci paper) that had shown that there is a large-scale bias for responses to oriented stimuli across V1. They demonstrated the responses to particular stimuli in voxels in $\mathrm{V} 1$ were correlated with the corresponding visual field angle for those voxels. They reported that the large-scale bias they had observed, rather than a smaller-scale bias introduced by the voxel sampling, was sufficient to drive the decoding of orientation information with multivariate methods (see also Beckett, Peirce et al. 2012, Wang, Merriam et al. 2014). In particular, we were interested to test how much data we would need to replicate the results from Freeman, Brouwer et al. (2011) when using high resolution 7T with dynamic shimming methods (described above) -3.375 $\mathrm{mm}^{3}$ compared to the previous study of $8 \mathrm{~mm}^{3}$. We were also interested to determine how the response amplitudes in visual cortex compared for retinotopic and "orientation" mapping stimuli at this higher spatial resolution, with a view to acquiring data at several different spatial resolutions to test whether the ratio between these two measures is independent of voxel size.

Three healthy subjects were scanned at $7 \mathrm{~T}$ using a 32-channel NOVA receive coil. We obtained written consent from the participants and the protocol was approved by the University of Nottingham ethics committee. One was scanned twice to assess repeatability. Each scanning session comprised two visual fMRI paradigms. The first paradigm was aimed at measuring cortical responses at the scale of retinotopic maps using a single, rotating wedge of a high-contrast checkerboard pattern. The second paradigm was aimed at measuring the signal at a much finer spatial scale due to the modulation of orientation-selective responses in early visual cortex. Note that recent experiments have shown that there is also an orientation dependent signal in early visual cortex at a larger spatial scale, reflecting a retinotopic bias for different orientations (Freeman, Brouwer et al. 2011). For this experiment, we used a single sinusoidal grating pattern $(0.75 \mathrm{cpd}$, full contrast) in an annulus (inner/outer radius $1^{\circ} / 6.1^{\circ}$, Gaussian blurred edge), that rotated around a central fixation cross in $24 \mathrm{~s}$. To control for drifts in attention, subjects performed fixation dimming task at threshold.

\section{[[ Figure 4 about here - vision results ]]}

2D GE-EPI BOLD fMRI data were collected using image-based shimming with the following parameters: FOV $192 \times 192 \mathrm{~mm}^{2}$, voxel size $1.5 \mathrm{~mm}$ isotropic, 34 slices, TE $25 \mathrm{~ms}$, TR $2000 \mathrm{~ms}$, flip angle $78^{\circ}$, BW $27.4 \mathrm{~Hz}, 96$ volumes acquired in $\sim 3.5$ minutes. For each subject, we obtained two retinotopic mapping scans and 11-14 orientation mapping scans and saved both modulus and phase data. $A B_{0}$-field map was formed from the average of $10 \mathrm{GE}$-EPI acquisitions collected prior to the 
fMRI paradigm at two echo times, $\mathrm{TE}_{1} 25 \mathrm{~ms}, \mathrm{TE}_{2} 28 \mathrm{~ms}$, but otherwise identical parameters as for the $\mathrm{fMRI}$ data. This allowed us to measure changes in the $B_{0}$-map over time and perform dynamic distortion correction allowing improved alignment to the anatomical data for visualizations on flat maps (see Methodological considerations). To aid registration of the $\mathrm{fMRI}$ data to anatomical images, we also obtained a $1.5 \mathrm{~mm}$ isotropic $\mathrm{T}_{1}$-weighted MPRAGE acquisition with the same slice prescription as the GE-EPI data. Additionally, for segmentation, cortical flattening and visualization, we obtained a T1-weighted whole-head MPRAGE in the same session with $1 \mathrm{~mm}$ isotropic voxels, 144 slices, TE $3.8 \mathrm{~ms}$, TR $15 \mathrm{~ms}$, flip angle $80^{\circ}$ (see Figure 1).

In related experiments, we also obtained anatomical data using: (1) a T2*-weighted 2D FLASH sequence for improved structural detail and to facilitate anatomical-functional registration $(0.5 \mathrm{~mm}$ in-plane resolution, $1.5 \mathrm{~mm}$ slice thickness, same slice position as the functional scans); (2) a wholehead, $\mathrm{T}_{1}$-weighted Phase-Sensitive Inversion Recovery (PSIR) anatomical scan, which allows correction of $B_{1}$-related inhomogeneities (Mougin, Abdel-Fahim et al. 2015). We used these data for cortical segmentation, inflation and flattening with the Freesurfer image analysis suite (http://surfer.nmr.mgh.harvard.edu/, Dale, Fischl et al. 1999).

We found large BOLD modulations in occipital cortex in response to retinotopic and orientation mapping stimuli (Figure 4A,B). Using standard analysis methods, we produced maps of visual field angle for each hemisphere in our subjects which were visualized on flattened representations of the occipital cortex. In each of the three subjects scanned, data from two 4 min scans were sufficient to produce robust visual field angle maps at $1.5 \mathrm{~mm}$ voxel resolution (Figure $4 \mathrm{C}$ ). Producing the corresponding map for the slowly rotating orientation stimulus required substantially more data, but showed a similar, large scale map structure. This was not surprising, because the signal modulations caused by the orientation stimulus were expected to be much smaller. A plot that relates statistical significance of responses to the amplitude of the $\mathrm{fMRI}$ responses in the orientation and retinotopy conditions can be used to further quantify this (Figure 5).

The large-scale spatial pattern of the orientation-specific response on the cortical surface appears to be sufficient to allow decoding of stimulus orientation from such visual cortex $\mathrm{fMRI}$ measurements

(Freeman, Heeger et al. 2013). We have not specifically addressed this question in the current study, but the maps in our sample of subjects suggest that the edges of the stimulus annulus may show particularly strong biases that can drive such results (see e.g. Figure 4D).

\section{[[Figure 5 about here]]}

To quantify the difference in responses to the two stimuli, we calculated a measure of BOLD CNR as the amplitude of the signal at the stimulus frequency $(1 / 24 \mathrm{~Hz}$ in both cases), normalized by an estimate of the noise floor - the combined signal in the top $1 / 3$ of high-frequency components of the time course. For the particular voxel size and acquisition scheme, the CNR for retinotopic scans far exceeded that for the more subtle orientation signal. For averages of two scans the CNR in the 
retinotopic scans was more than 3 times higher, and even with an average of 14 scans, seven times more data, the CNR for the orientation scans reached only about 50\% (Figure 4E). There was a strong correlation between the visual field angle preference and the preferred orientation for each voxel in V1 (Figure 4F). The scatter plots show data for ventral and dorsal V1 of an example subject but the same correlation was apparent in all subjects across all of V1-V3 (see Supplementary Table 1). Interestingly, the correlation between preferred visual field angle and orientation was consistently lower for the ventral sub-divisions of the visual areas. This may reflect the local anatomy of more ventrally located visual areas, in particular the proximity of large dural veins (Winawer, Horiguchi et al. 2010).

\section{Discussion}

In our experiments, we were particularly interested in determining the detailed function in individual subjects and relating this to their anatomy, with a view towards translational and potentially clinically applications. Here we demonstrate, as do other neuroscience labs, how to address basic functional mechanisms in smaller groups of subjects first - using many, repeated measurements in individuals before moving on to larger group studies. The techniques and analysis may be applied to the study of other functional areas in the brain, for example the auditory cortex (Besle, Mougin et al. 2015), where functional and structural MRI at $7 \mathrm{~T}$ has been used for measuring tonotopy, frequency selectivity as well as anatomical markers (myelination) to delineate auditory fields in individual subjects. In the clinical setting, tumours, abnormal anatomy, tissue changes due to stroke or surgery can lead substantially to the between-subject variability. Using high spatial resolution UHF functional and structural imaging techniques these questions can now be probed.

For many neuroscience applications, fMRI at UHF has clear advantages. In developing new acquisition methods and paradigms, testing against a ground-truth and previous results, and determining reproducibility is important. Here, we show how fMRI of somatosensory and visual cortex can be used to improve our understanding of the function and layout of these areas in the human brain. With robust measurements at voxel sizes of $1 \mathrm{~mm}^{3}$ and below, spatially resolving small brain structures has become possible. For example, we have used 3D-EPI to acquire high-resolution fMRI data in visual cortex with volume receive coils (Schluppeck D Merriam E 2010, Goncalves, Ban et al. 2015) and are now testing data acquisitions in somatosensory cortex with local surface receive coils (2D-EPI) as shown here. The improved BOLD CNR at $7 \mathrm{~T}$ has made measuring weaker signals, such as biases across cortical maps easier in individual participants (Beckett, Peirce et al. 2012). At the same time, the sensitivity of functional signals from methods other than gradient-echo EPI are more robust and usable.

Overall, we believe that with continued technical developments, more powerful analysis frameworks and computing power, neuroimaging at $7 \mathrm{~T}$ is becoming increasingly appealing to neuroscientists. There is often a difference in the approach to research problems between scientists from different 
backgrounds, with the emphasis of MR physicists often on establishing and understanding the feasibility of a particular technique, whilst neuroscientists may be driven more by a desire for stability at the cost of technical improvements, with consistency of results usually more important than pushing the boundaries of the data acquisition scheme. We hope that our results show the potential advancements in mapping somatosensory and visual areas using high spatial resolution UHF fMRI, and provide an example of how scientists across interdisciplinary fields can make progress together without compromises. 


\section{Figures}

\section{Figure 1 - Methodology}

(A) Diagram illustrating data processing and image registration steps for visualizing statistical maps from high spatial resolution $\mathrm{fMRI}$ experiments on anatomical images, and the derived inflated or flattened cortical surface representations. (B) Example anatomical image illustrating some of the data used in our experiments. Shown is a phase-sensitive inversion recovery (PSIR) image acquired with $0.3 \mathrm{~mm}$ in-plane spatial resolution and $1 \mathrm{~mm}$ slice thickness.

\section{Figure 2 - Imaging somatosensory cortex}

Summary of somatosensory cortex imaging results (replotted from Sanchez-Panchuelo et al, 2012). (A) Reconstructed right cortical surface of one subject in grayscale (dark gray sulci, light gray gyri). Color overlay shows stimulus preference - stimulation at which finger tip led to the largest response. (B) Flattened representation of area around central sulcus, including pre- and post-central gyrus. There is a smooth progression of stimulus preference from little finger to thumb mapped onto the cortical surface in the superior-inferior direction along the bank of the post-central gyrus (C) Zoomedin illustration of the map of digit representation for this subject. Black line, area of cortex representing index finger, the region of interest for additional sets of measurements. (D) Location of vibrotactile stimuli on the index finger. This experiment allows subdivision of $\mathrm{S} 1$ finger-maps based on the known layout of adjacent maps in cytoarchitectonic subregions of S1 (see Sanchez-Panchuelo, Besle et al. (2012) for details). (E) Areas of S1 responding to periodic stimulation of sites in D. Color map, coherence with best-fitting sinusoid at the frequency of periodic stimulation $(1 / 30 \mathrm{~Hz})(\mathrm{F})$ Mapping of the index finger surface (proximal to distal) on the cortical surface for the same subject. Colors show the stimulus preference as quantified by response phase in a periodic stimulation paradigm. Colorbar indicates correspondence between phase values and stimulation site on index finger.

\section{Figure 3 - Data acquisition with different protocols and coils}

(A) Temporal SNR maps for GE-EPI data obtained with a 32-channel volume transmit-receive coil (Nova Medical). Temporal SNR is high in all areas of cortex. Voxel size,1.5 mm isotropic (voxel volume, $3.375 \mathrm{~mm}^{3}$ ); TR $2 \mathrm{~s}$, TE $28 \mathrm{~ms}$. Color bar indicates temporal SNR values. (B) Measurements with a local surface receive coil (32-channel, MRCoils) provide improved temporal SNR close to the 
surface, allowing for higher spatial resolution acquisition. Despite the voxel volumes being more than $5 \times$ smaller, they provide good signal and tSNR for fMRI measurements. Voxel size, $0.8 \mathrm{~mm}$ in-plane, $1 \mathrm{~mm}$ slice thickness (voxel volume, $0.64 \mathrm{~mm}^{3}$ ). Color scale is the same as in A. (C) Results from a digit somatotopy experiment on a flattened patch of the cortical surface. Data are from an example subject obtained with $1.5 \mathrm{~mm}$ isotropic $\left(3.375 \mathrm{~mm}^{3}\right)$ voxels using the volume receive coil. Total acquisition time: $8 \mathrm{~min}$. Grayscale, gyri and sulci as in Figure 2. Colors, phase values representing preferred stimulation site on the hand as indicated by the colorbar. Statistical maps threshold using the TFCE method (Smith and Nichols 2009). For parameter choices see Sanchez-Panchuelo, Francis et al. (2010). (D) Data obtained with the same stimuli from the same subject - but with the local surface receive coil and $0.64 \mathrm{~mm}^{3}$ voxels. Total acquisition time: $12 \mathrm{~min}$. Plotting conventions and statistical thresholding as in $\mathrm{C}$. The map provides much finer grained detail about the exact layout of S1.

(E) Functional MR images from the digit mapping experiment. Grayscale image, GE-EPI image (averaged over 60 time points). At this high resolution, high-quality fMRI data itself can be used to provide useful anatomical information, including about the gray matter/white matter boundary.

\section{Figure 4 - Visual stimuli and data}

(A) Retinotopic stimuli for measuring visual field angle. This provides a very strong, robust signal. A wedge of high-contrast checkerboards moved through the visual field in $24 \mathrm{~s}$. To ensure stable fixation and to control the attentional state, subjects performed a dimming task on the fixation cross at the center of the screen (yellow cross). (B) Orientation mapping stimulus. Responses to this stimulus are more subtle and there is a debate about whether they are driven by uneven sampling of orientation columns or large-scale biases across visual cortex. A high-contrast sinusoidal grating $(0.75 \mathrm{cpd})$ rotated through $180^{\circ}$ in $24 \mathrm{~s}$. The stimulus was windowed by an annulus (inner/outer

radius, $1^{\circ} / 6.1^{\circ}$, outer edge blurred). Subjects performed the same task as with the retinotopic stimuli.

(C) Example retinotopic map of angular position on flattened area around occipital pole (right hemisphere, example subject 2). Dark gray indicates sulci and light gray gyri. Colors, preferred visual field angle inferred from the phase of $\mathrm{FMRI}$ response. Red corresponds to upper vertical meridian, green to horizontal meridian, and magenta to the lower vertical meridian. Note that the color map ( $h s v$ ) is scaled to cover one visual hemifield $\left(0^{\circ}\right.$ to $180^{\circ}$ of visual field angle), to facilitate comparison with the map obtained for orientation. 
(D) Example map of preferred orientation. Same subject, anatomical area and display conventions as in C. Colors here indicate the preferred stimulus orientation - from $0^{\circ}$ to $180^{\circ}-$ inferred from phase of the $\mathrm{fMRI}$ response.

Inset images in $C$ and $D$ show an example slice from the GE-EPI data set (gray scale) and superimposed, corresponding statistical maps (blue-red) indicating areas at the occipital pole with reliable modulation (coherence, $c>0.3$ ) at the stimulus frequency $(1 / 24 \mathrm{~Hz})$.

(E) Comparison of the BOLD contrast-to-noise for the two different paradigms. Plot shows mean BOLD CNR measures across subjects as a function of number of scans averaged (error bars indicate SEM across $n=3$ subjects). Although the absolute value of CNR in the orientation scans is nearly 10 with an average of two to four 4 min scans, the signal in the retinotopy scans (red symbol) exceeds them by a factor of 3-4.

(F) Scatter plot of the preferred stimulus orientation as a function of retinotopic location (both quantified by response phase in the periodic design). The plot shows data for the same subject as in panels $C$ and D. Green symbols, voxels in the ventral portion of V1, representing the upper visual field quadrant. Blue symbols, dorsal V1, representing the lower visual field quadrant. There is a clear correlation between the visual field angle and the preferred stimulus orientation for this subject (V1v: Pearson's $r=0.35, p \approx 0, n=574$ voxels; $V 1 d: r=0.62, p \approx 0), n=520$ voxels).

\section{Figure 5 - Visual stimuli and data}

Plot of $\mathrm{fMRI}$ signal coherence with stimulus against $\mathrm{fMRI}$ response amplitude (in \% signal change) from V1 in an example subject. Red symbols, data for retinotopy scan; black symbols, data for orientation stimulus. Gray and pink lines shows a moving average of the orientation and retinotopy data, respectively (Savitzky-Golay, span 121 data points). Response amplitudes in the retinotopy data (red, closed symbols) are larger - up to $10 \%$ signal change - than for the orientation stimulus (black, open symbols). Also, generally, higher coherence values are reached for the retinotopy data. Larger coherence values are reached for the orientation stimuli at the same amplitude of fMRI response (left-ward shift of the gray curve), because for this subject, the orientation data was averaged over 12 runs, the retinotopy data only over 2 runs. Note the logarithmic scale on the $x$ axis. 


\section{References}

Adams, D. L., L. C. Sincich and J. C. Horton (2007). "Complete pattern of ocular dominance columns in human primary visual cortex." Journal of Neuroscience 27(39): 10391-10403.

Andersson, J. L., S. Skare and J. Ashburner (2003). "How to correct susceptibility distortions in spin-echo echo-planar images: application to diffusion tensor imaging." Neuroimage 20(2): 870888.

Bandettini, P. A. (2012). "Twenty years of functional MRI: the science and the stories." Neuroimage 62(2): 575-588.

Barry, R. L., S. C. Strother and J. C. Gore (2012). "Complex and magnitude-only preprocessing of 2D and 3D BOLD fMRI data at 7 T." Magn Reson Med 67(3): 867-871.

Beckett, A., J. W. Peirce, R. M. Sanchez-Panchuelo, S. Francis and D. Schluppeck (2012). "Contribution of large scale biases in decoding of direction-of-motion from high-resolution fMRI data in human early visual cortex." Neuroimage 63(3): 1623-1632.

Besle, J., O. Mougin, R. Sanchez-Panchuelo, Gowland P, Bowtell R, Francis S and Krumbholz K (2015). Structural and functional mri mapping of human auditory cortex at $7 \mathrm{t}$ and its possible application to measuring cortical re-organization in hearing-impairment and tinnitus. . 9th TRI Tinnitus Conference, Ann Arbor, MI, USA.

Besle, J., R. M. Sanchez-Panchuelo, R. Bowtell, S. Francis and D. Schluppeck (2013). "Singlesubject fMRI mapping at $7 \mathrm{~T}$ of the representation of fingertips in S1: a comparison of event-related

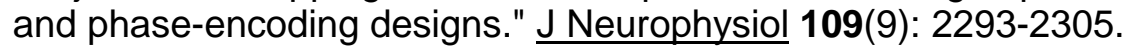

Besle, J., R. M. Sanchez-Panchuelo, R. Bowtell, S. Francis and D. Schluppeck (2014). "Eventrelated fMRI at 7T reveals overlapping cortical representations for adjacent fingertips in $\mathrm{S} 1$ of individual subjects." Hum Brain Mapp 35(5): 2027-2043.

Budinger, T. F., M. D. Bird, L. Frydman, J. R. Long, T. H. Mareci, W. D. Rooney, B. Rosen, J. F. Schenck, V. D. Schepkin, A. D. Sherry, D. K. Sodickson, C. S. Springer, K. R. Thulborn, K. Ugurbil and L. L. Wald (2016). "Toward $20 \mathrm{~T}$ magnetic resonance for human brain studies: opportunities for discovery and neuroscience rationale." MAGMA 29(3): 617-639.

Dale, A. M., B. Fischl and M. I. Sereno (1999). "Cortical surface-based analysis. I. Segmentation and surface reconstruction." Neuroimage 9(2): 179-194.

De Martino, F., J. Zimmermann, L. Muckli, K. Ugurbil, E. Yacoub and R. Goebel (2013). "Cortical depth dependent functional responses in humans at 7T: improved specificity with 3D GRASE." PLoS One 8(3): e60514.

Dumoulin, S. O. and B. A. Wandell (2008). "Population receptive field estimates in human visual cortex." Neuroimage 39(2): 647-660.

Feinberg, D. A., S. Moeller, S. M. Smith, E. Auerbach, S. Ramanna, M. F. Glasser, K. L. Miller, K. Ugurbil and E. Yacoub (2010). "Multiplexed Echo Planar Imaging for Sub-Second Whole Brain FMRI and Fast Diffusion Imaging." Plos One 5(12).

Francis, S. T., E. F. Kelly, R. Bowtell, W. J. Dunseath, S. E. Folger and F. McGlone (2000). "fMRI of the responses to vibratory stimulation of digit tips." Neuroimage 11(3): 188-202. 
Freeman, J., G. J. Brouwer, D. J. Heeger and E. P. Merriam (2011). "Orientation decoding depends on maps, not columns." J Neurosci 31(13): 4792-4804.

Freeman, J., D. J. Heeger and E. P. Merriam (2013). "Coarse-scale biases for spirals and orientation in human visual cortex." J Neurosci 33(50): 19695-19703.

Goncalves, N. R., H. Ban, R. M. Sanchez-Panchuelo, S. T. Francis, D. Schluppeck and A. E. Welchman (2015). "7 tesla FMRI reveals systematic functional organization for binocular disparity in dorsal visual cortex." $\mathrm{J}$ Neurosci 35(7): 3056-3072.

Harel, N. (2012). "Ultra high resolution fMRI at ultra-high field." Neuroimage 62(2): 1024-1028.

Harmer, J., S. Francis and R. Bowtell (2011). Dynamic Distortion Correction of SE EPI data using Phase Maps from Simultaneously-Acquired GE-EPI data. ISMRM Abstracts, Montreal

Haynes, J. D. and G. Rees (2005). "Predicting the orientation of invisible stimuli from activity in human primary visual cortex." Nat Neurosci 8(5): 686-691.

Huber, L., J. Goense, A. J. Kennerley, R. Trampel, M. Guidi, E. Reimer, D. Ivanov, N. Neef, C. J. Gauthier, R. Turner and H. E. Moller (2015). "Cortical lamina-dependent blood volume changes in human brain at 7 T." Neuroimage 107: 23-33.

Huber, L., D. Ivanov, S. N. Krieger, M. N. Streicher, T. Mildner, B. A. Poser, H. E. Moller and R. Turner (2014). "Slab-selective, BOLD-corrected VASO at 7 Tesla provides measures of cerebral blood volume reactivity with high signal-to-noise ratio." Magn Reson Med 72(1): 137-148.

Hutton, C., A. Bork, O. Josephs, R. Deichmann, J. Ashburner and R. Turner (2002). "Image distortion correction in fMRI: A quantitative evaluation." Neuroimage 16(1): 217-240.

Kamitani, Y. and F. Tong (2005). "Decoding the visual and subjective contents of the human brain." Nat Neurosci 8(5): 679-685.

Moeller, S., E. Yacoub, C. A. Olman, E. Auerbach, J. Strupp, N. Harel and K. Ugurbil (2010). "Multiband Multislice GE-EPI at 7 Tesla, With 16-Fold Acceleration Using Partial Parallel Imaging With Application to High Spatial and Temporal Whole-Brain FMRI." Magnetic Resonance in Medicine 63(5): 1144-1153.

Mougin, O., R. Abdel-Fahim, R. Dineen, A. Pitiot, N. Evangelou and P. Gowland (2015). "Imaging gray matter with concomitant null point imaging from the phase sensitive inversion recovery sequence." Magn Reson Med.

Mountcastle, V. B. (1997). "The columnar organization of the neocortex." Brain 120: 701-722.

Nelson, A. J. and R. Chen (2008). "Digit somatotopy within cortical areas of the postcentral gyrus in humans." Cereb Cortex 18(10): 2341-2351.

Norris, D. G. (2012). "Spin-echo fMRI: The poor relation?" Neuroimage 62(2): 1109-1115.

Olman, C. A., N. Harel, D. A. Feinberg, S. He, P. Zhang, K. Ugurbil and E. Yacoub (2012). "Layerspecific fMRI reflects different neuronal computations at different depths in human V1." PLoS One 7(3): e32536.

Overduin, S. A. and P. Servos (2008). "Symmetric sensorimotor somatotopy." PLoS One 3(1): e1505.

Penfield, W. and E. Boldrey (1937). "Somatic motor and sensory representation in the cerebral cortex of man as studied by electrical stimulation." Brain 60: 389-443. 
Poole, M. and R. Bowtell (2008). "Volume parcellation for improved dynamic shimming." MAGMA 21(1-2): 31-40.

Poser, B. A., P. J. Koopmans, T. Witzel, L. L. Wald and M. Barth (2010). "Three dimensional echoplanar imaging at 7 Tesla." Neuroimage 51(1): 261-266.

Sanchez Panchuelo, R. M., R. Ackerley, P. M. Glover, R. W. Bowtell, J. Wessberg, S. T. Francis and F. McGlone (2016). "Mapping quantal touch using 7 Tesla functional magnetic resonance imaging and single-unit intraneural microstimulation." Elife 5.

Sanchez Panchuelo, R. M., D. Schluppeck, J. Harmer, R. Bowtell and S. Francis (2015).

"Assessing the spatial precision of SE and GE-BOLD contrast at 7 Tesla." Brain Topogr 28(1): 6265.

Sanchez-Panchuelo, R. M., J. Besle, A. Beckett, R. Bowtell, D. Schluppeck and S. Francis (2012). "Within-digit functional parcellation of brodmann areas of the human primary somatosensory cortex using functional magnetic resonance imaging at 7 tesla." $\underline{\mathrm{J} \text { Neurosci }}$ 32(45): 15815-15822.

Sanchez-Panchuelo, R. M., J. Besle, O. Mougin, P. Gowland, R. Bowtell, D. Schluppeck and S. Francis (2014). "Regional structural differences across functionally parcellated Brodmann areas of human primary somatosensory cortex." Neuroimage 93 Pt 2: 221-230.

Sanchez-Panchuelo, R. M., S. Francis, R. Bowtell and D. Schluppeck (2010). "Mapping human somatosensory cortex in individual subjects with 7T functional MRI." J Neurophysiol 103(5): 25442556.

Schluppeck D Merriam E, S. P. R., Francis S, Bowtell R, Velasco P, Inati S, Heeger DJ (2010). "Assessing the spatial precision of high-resolution echo-planar functional MRI at 3T and 7T." 2010 Neuroscience Meeting Planner. San Diego, CA, 483.5/TT15.

Siero, J. C., N. F. Ramsey, H. Hoogduin, D. W. Klomp, P. R. Luijten and N. Petridou (2013). "BOLD specificity and dynamics evaluated in humans at $7 \mathrm{~T}$ : comparing gradient-echo and spinecho hemodynamic responses." PLoS One 8(1): e54560.

Smith, S. M. and T. E. Nichols (2009). "Threshold-free cluster enhancement: addressing problems of smoothing, threshold dependence and localisation in cluster inference." Neuroimage 44(1): 8398.

Ugurbil, K. (2012). "The road to functional imaging and ultrahigh fields." Neuroimage 62(2): 726735.

van der Zwaag, W., R. Kusters, A. Magill, R. Gruetter, R. Martuzzi, O. Blanke and J. P. Marques (2012). "Digit somatotopy in the human cerebellum: A 7T fMRI study." Neuroimage.

Wandell, B. A., S. O. Dumoulin and A. A. Brewer (2007). "Visual field maps in human cortex." Neuron 56(2): 366-383.

Wang, H. X., E. P. Merriam, J. Freeman and D. J. Heeger (2014). "Motion direction biases and decoding in human visual cortex." J Neurosci 34(37): 12601-12615.

Wilson, J. L., M. Jenkinson and P. Jezzard (2002). "Optimization of static field homogeneity in human brain using diamagnetic passive shims." Magn Reson Med 48(5): 906-914.

Winawer, J., H. Horiguchi, R. A. Sayres, K. Amano and B. A. Wandell (2010). "Mapping hV4 and ventral occipital cortex: the venous eclipse." $\underline{\mathrm{J} \text { Vis } 10(5): 1 .}$ 
Yacoub, E., N. Harel and K. Ugurbil (2008). "High-field fMRI unveils orientation columns in humans." Proceedings of the National Academy of Sciences of the United States of America 105(30): 10607-10612.

Yacoub, E., A. Shmuel, N. Logothetis and K. Ugurbil (2007). "Robust detection of ocular dominance columns in humans using Hahn Spin Echo BOLD functional MRI at 7 Tesla." Neuroimage 37(4): 1161-1177. 
raw GE-EPI data

[mag / ph

reconstructed]<smiles>C=[V]</smiles>

dynamic distortion

correction

[SPHERE]<smiles>C1CCC1</smiles>

motion correction

[within / between

scans]

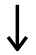

temporal high pass filtering, drift correction

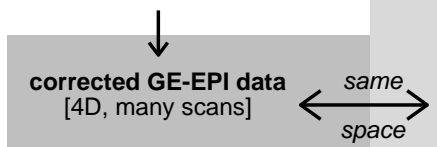

constant across sessions

flat maps

mrFlatMesh

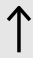

cortical segmentation [white matter, pial]<smiles>CCCC</smiles>

whole head anatomy [MPRAGE or PSIR]

$$
\text { 个 same contrast }
$$

in-plane MPRAGE

个 same orientation

reference GE-EPI image [3D, average across a scan]

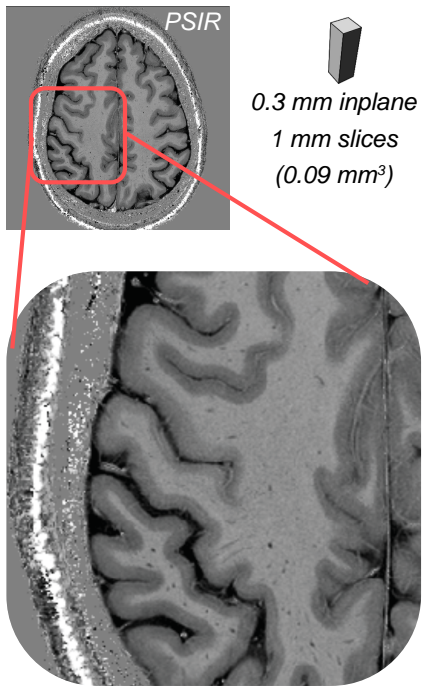



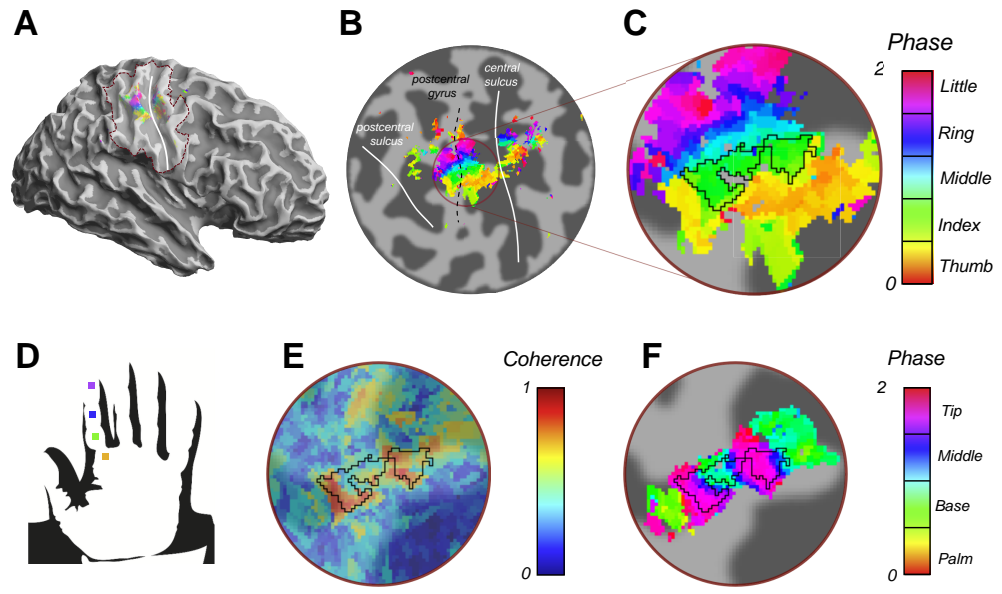
A

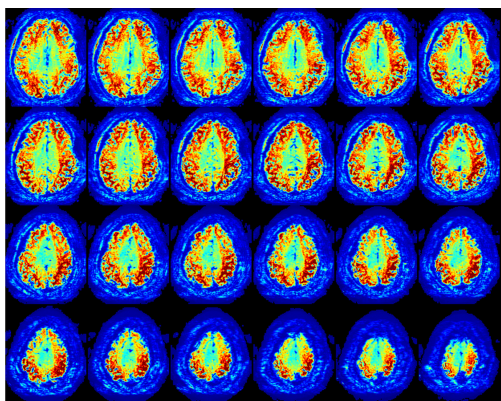

C

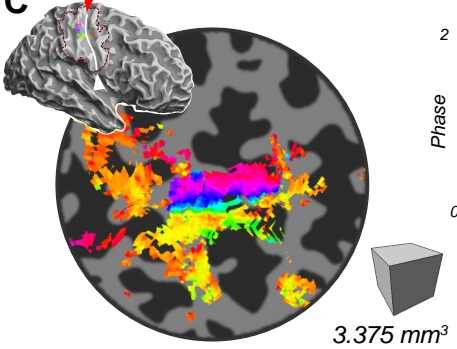

B
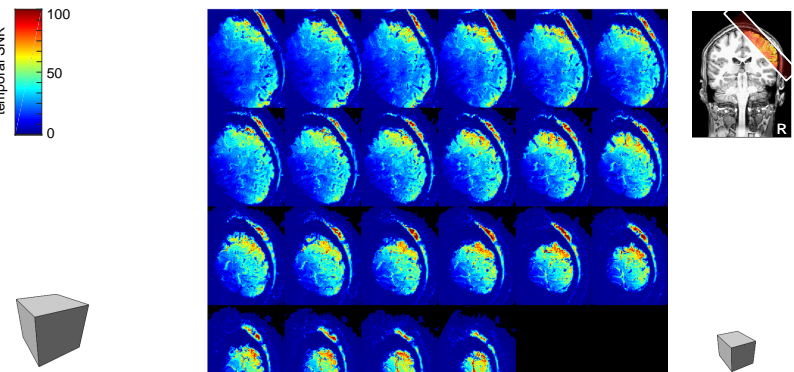

$3.375 \mathrm{~mm}^{3}$

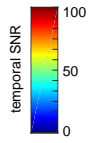

$\nabla$

i)

$0.64 \mathrm{~mm}^{3}$

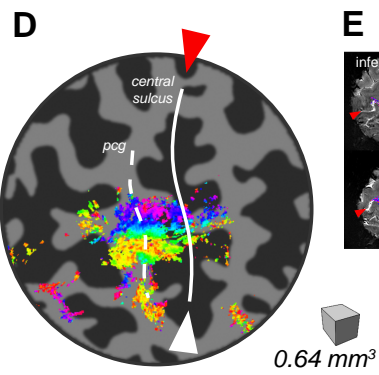

E

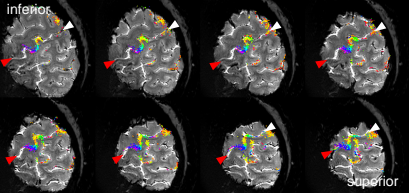


A retinotopy

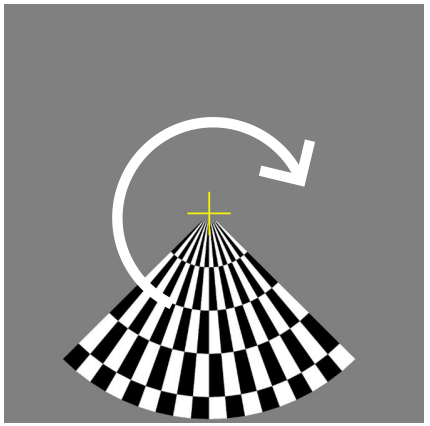

C

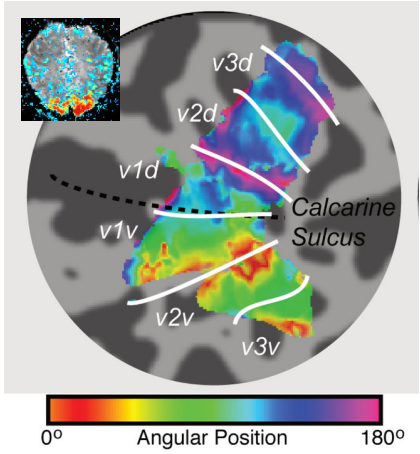

E $\quad 31.53+\oint \quad \begin{gathered}\text { retinotopy } \\ \text { scans }\end{gathered}$

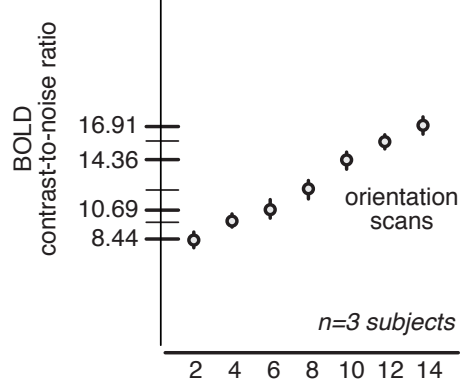
Number of averaged scans
B orientation

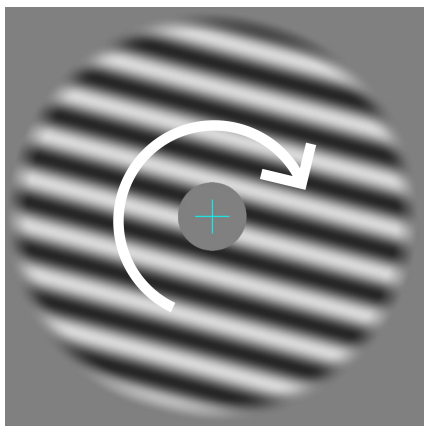

D

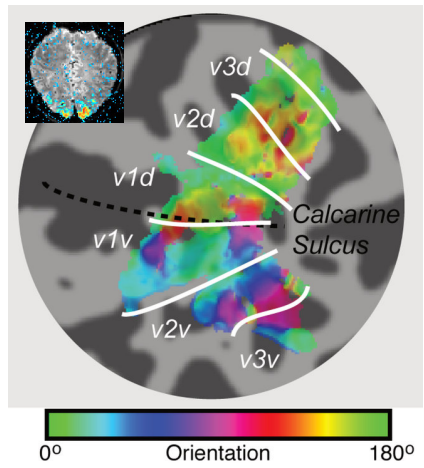

$\mathbf{F}$

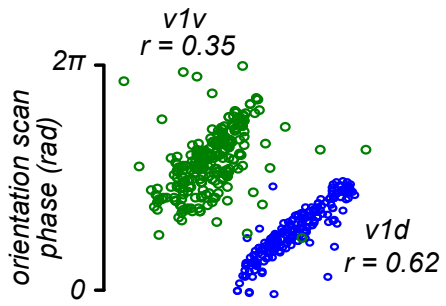

subject 2

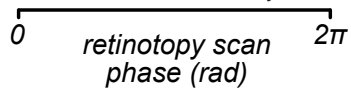


A

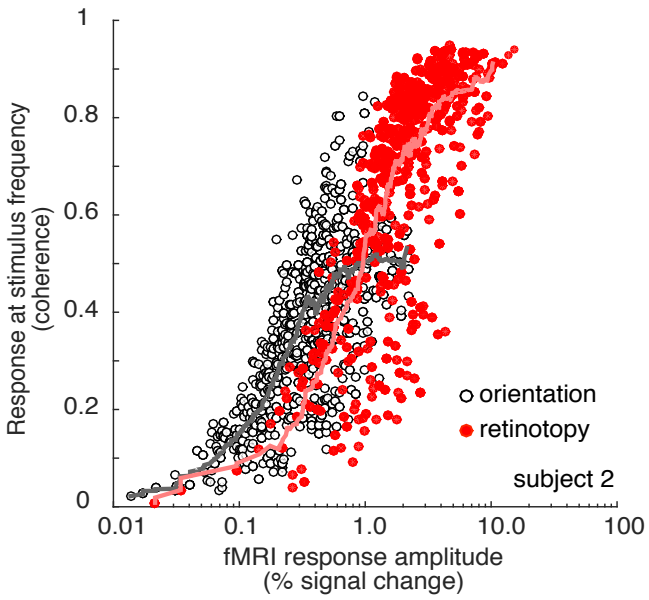

